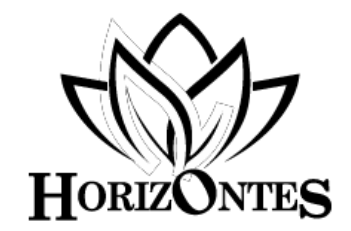

Horizontes. Revista de Investigación en Ciencias de la Educación Https://doi.org/10.33996/revistahorizontes.v2i6.47 abril - junio, 2018 Volumen 2 / No. 6

www.revistahorizontes.org pp. $73-89$

\title{
El liderazgo distribuido y la percepción de los equipos directivos de las escuelas públicas de educación primaria madrileñas
}

\author{
The distributed leadership and the perception of the public schools of from \\ Madrid directors team
}

\author{
Ingrid del Valle García Carreño \\ ivgarcar@alu.upo.es
}

Universidad Pablo de Olavide, Sevilla, España

\section{RESUMEN}

La presente investigación tuvo como objetivo caracterizar el ejercicio del liderazgo distribuido de los directivos de las escuelas públicas de Madrid, en cada una de sus dimensiones. Se trabajó desde la comprensión holística de la ciencia. El tipo de investigación fue descriptiva, se caracterizó el ejercicio del LD en cada una de sus cuatro dimensiones, se precisó la sinergia que se establece entre ellas, en función de distintas variables de contexto. El diseño fue de campo, transeccional y contemporáneo. La muestra fue de 71 escuelas, 132 directivos: 71 directores y 61 jefes de estudio. Los datos se recogieron mediante la Escala de Liderazgo distribuido, con un índice de validez de 0,75 y una confiabilidad de Alfa de Cronbach de 0,86 . De igual manera el estudio mostró la tendencia de los directivos a interactuar, con los líderes formales, informales, los seguidores y los diferentes actores pertenecientes al contexto de la escuela, para propiciar la distribución social de la influencia en el grupo; y delegaban las funciones de dirección, orientación, la toma de decisiones, las tareas y los compromisos que guían y dan sentido a la organización.

Palabras clave: Dirección escolar, Escuelas públicas de Madrid, Liderazgo distribuido, Liderazgo educativo

\begin{abstract}
The purpose of this research was to characterize the exercise of distributed leadership of the directors of the public schools of Madrid, in each of its dimensions. It worked from the holistic understanding of science. The type of research was descriptive, the exercise of ML was characterized in each of its four dimensions, and the synergy established between them was specified, depending on different context variables. The design was field, transectional and contemporary. The sample consisted of 71 schools, 132 executives: 71 directors and 61 heads of study. Data were collected using the Distributed Leadership Scale, with a validity index of 0.75 and a Cronbach Alpha reliability of 0.86 . Similarly, the study showed the tendency of managers to interact, with formal, informal leaders, followers and the different actors belonging to the context of the school, to promote the social distribution of influence in the group; and delegated the functions of direction, orientation, decision making, tasks and commitments that guide and make sense of the organization.
\end{abstract}

Key words: School management, Public schools of Madrid, Distributed leadership, Educational leadership 


\section{INTRODUCCIÓN}

La escuela del siglo XXI se enfrenta a una sociedad donde el cambio se ha convertido en una prioridad permanente, influenciada por la tecnología, los acelerados procesos de globalización, mundialización, cambios de leyes y programas, innumerables conflictos sociales y currículos, inmigración descontrolada y desigualdades. El liderazgo distribuido (LD) es un concepto emergente relevante para la cultura del sector educativo, es una forma de liderazgo que se sustenta en una filosofía más colectiva e incluyente que la teoría del liderazgo tradicional, se centra en las habilidades, rasgos y comportamientos de los individuos como líderes.

A nivel internacional, varios países están involucrados en el estudio del estilo de liderazgo distribuido: (i) la perspectiva británica expresa que el liderazgo distribuido, se ha centrado en conceptualizaciones teóricas en los distintos niveles educativos (Harris y Spillane, 2008; Bolden, 2011; Day et al., 2011); (ii) la posición en Australia, acerca de este liderazgo distribuido se ha explorado en niveles de secundaria y superior (Gronn, 2002, 2006; Gronn y Hamilton, 2004; Dinham et al., 2011; Sloan, 2013); (iii) en EE.UU, una estrategia que se caracteriza por ir hacia la mejora de la calidad de liderazgo, de la escuela para garantizar a las instituciones educativas líderes y el trabajo de manera efectiva, en el liderazgo múltiple o liderazgo distribuido (Centre for School Improvement 2001, 2003 como se citó en Oduro, 2004).

Al concepto no se le había dado mucha importancia hasta hace pocos años, cuando el National College for School Leadership (NCSL, 2008), retomó el discurso y lo colocó como un principio esencial de la literatura sobre el desarrollo del liderazgo escolar (MacBeath et al, 2004; Spillane, 2005; Leithwood, 2009). Sin embargo, existe un creciente reconocimiento de las prácticas del liderazgo distribuido, éstas han ido en aumento y van más allá de la teorización de las prácticas de dicho liderazgo. Existen varias investigaciones que avalan este liderazgo, el cual se caracteriza por no centralizar el poder y por distribuir los roles de autoridad entre múltiples seguidores (Gronn, 2000; Harris, 2004; Spillane et al., 2004; Gunter y Fitzgerald, 2008). El liderazgo distribuido ha reconocido efectos significativos sobre la calidad de las escuelas, ha generado importantes cambios, en la visión de cómo se distribuye y delega la responsabilidad en las escuelas y también en los beneficios y crecimiento, sugeridos en el uso y desarrollo del mismo (Spillane, 2006).

En esta investigación se siguió el criterio desarrollado por Bolden, (2011), el cual se basa específicamente en los cuatro autores destacados en este tipo de liderazgo: Gronn (2002); MacBeath et al. (2004); Spillane (2005); Leithwood (2009). Se estudiaron cuatro dimensiones del liderazgo distribuido (visión, misión y metas compartidas, prácticas de liderazgo distribuido, desarrollo del personal, rediseño de la organización), con esto se trató, de definir cómo las dimensiones influían en el trabajo de los directivos, así como su impacto en la organización y la percepción del mismo.

La gestión escolar se convierte así en una encrucijada entre lo burocrático, lo administrativo, lo económico, lo político, lo ideológico, lo pedagógico y lo psicológico, es decir, abarca toda dimensión organizativa, pero a diferencia del gestor, el líder, además de realizar todas estas funciones, dinamiza, ilusiona, arrastra en la construcción y reconstrucción permanente de un proyecto educativo.

El liderazgo escolar se ha convertido en una prioridad de los programas de política educativa a nivel internacional (Pont et al., 2008), por ello, la posición del director 
constituye un factor esencial que se enfrenta a una serie de retos, debido a que en los últimos años ha cambiado la visión de lo que las escuelas deben lograr, puesto que los países necesitan desarrollar nuevas formas de liderazgo escolar para mejorar los actuales y futuros contextos educativos.

Para Gairín y Muñoz (2013), la función directiva $y$ el liderazgo educativo son fundamentales para la creación de la visión del centro, la cual debe ser compartida por el profesorado y por toda la comunidad educativa. Tanto el aprendizaje del alumnado, como la calidad de la escuela requieren de una participación activa de los docentes, el resto de la comunidad educativa es im-prescindible para que la dirección asuma roles de cohesión del grupo humano, coordinación y animación de la participación.

En este sentido, el liderazgo distribuido proporciona un nuevo marco conceptual que posibilita reconceptualizar y reconfigurar las prácticas del liderazgo en las escuelas (Harris, 2004, 2014; Harris et al., 2013).

Los líderes tienden a tomar iniciativas, $\mathrm{y}$ en las escuelas eficaces, los directores enfatizan en la selección, contratación y el desarrollo del personal en servicio, así como en la importancia del consenso, la consistencia, la calidad de la enseñanza, la constancia del comportamiento y las prácticas del personal (Barber y Mourshed, 2007).

Sin embargo, en la práctica, los líderes escolares no pueden tener un verdadero impacto en los resultados del estudiante si no cuentan con una autonomía y apoyo suficientes para tomar decisiones importantes, y si sus principales responsabilidades están bien definidas y centradas en la enseñanza y el aprendizaje (Pont et al., 2008).

Por último la investigación tuvo está enmarcada en mejorar el trabajo de los directores es importante saber qué hacen para poder identificar sus actuaciones y así evaluar la incidencia positiva en el desempeño de los estudiantes. Manteniendo el como objetivo la caracterización en el ejercicio del liderazgo distribuido de los directivos de las escuelas públicas de Madrid, en cada una de sus dimensiones.

\section{MÉTODO}

Para el desarrollo de la investigación se realizó un estudio descriptivo, desde una compresión holística de la ciencia, con un diseño de campo, transeccional y contemporáneo. La población estuvo formada por los directivos de las 243 escuelas públicas de Madrid. Se hizo un muestreo probabilístico y la muestra fue de 71 escuelas, en las cuales había 132 directivos: 71 directores y 61 jefes de estudio. El 54\% de los directivos eran mujeres y el $46 \%$ hombres, con edades entre 39 y 63 años de edad.

Para recoger los datos, se aplicó a los directivos la Escala de Liderazgo distribuido, con una confiabilidad Alfa de Cronbach de 0,86 . El instrumento midió las cuatro dimensiones del evento: Prácticas de liderazgo distribuido con 7 ítems, Decisiones compartidas en la organización con 14 ítems, Misión, visión y metas compartidas con 11 ítems, y Desarrollo profesional y complejidad de la tarea con 13 ítems.

Como técnica de análisis, se utilizó la estimación de la mediana, se calcularon los cuartiles, el máximo y el mínimo, y se representaron con gráficos de caja y bigote, para visualizar la distribución de los datos y para analizar los casos atípicos. Asimismo, se calcularon las frecuencias y porcentajes de escuelas por cada categoría de interpretación y se ilustró la información con un gráfico de sectores. 
RESULTADOS intervalos de interpretación. El resultado

La escala de Liderazgo distribuido está global del liderazgo distribuido ejercido por diseñada sobre un puntaje bruto, de liderazgo distribuido, de 180 puntos, que se transformó a una escala de 50 puntos para facilitar el análisis descriptivo. Los puntajes de cada dimensión también fueron transformados a una escala de 50 puntos y se utilizaron cinco los directivos en las escuelas obtuvo una mediana de 34,76 puntos sobre una escala de 50. Esta mediana se ubicó en la categoría alta, lo que significa que en términos generales el liderazgo distribuido estaba presente en las escuelas estudiadas (ver Tabla 1)

Tabla 1. Mediana del liderazgo distribuido

\begin{tabular}{lll}
\hline Mediana & & $\mathbf{3 4 , 7 6}$ \\
\hline Mínimo & & 25,28 \\
Máximo & & 47,04 \\
Percentiles & 25 & 32,79 \\
& 50 & 34.76 \\
\hline & 75 & 36,94 \\
\hline
\end{tabular}

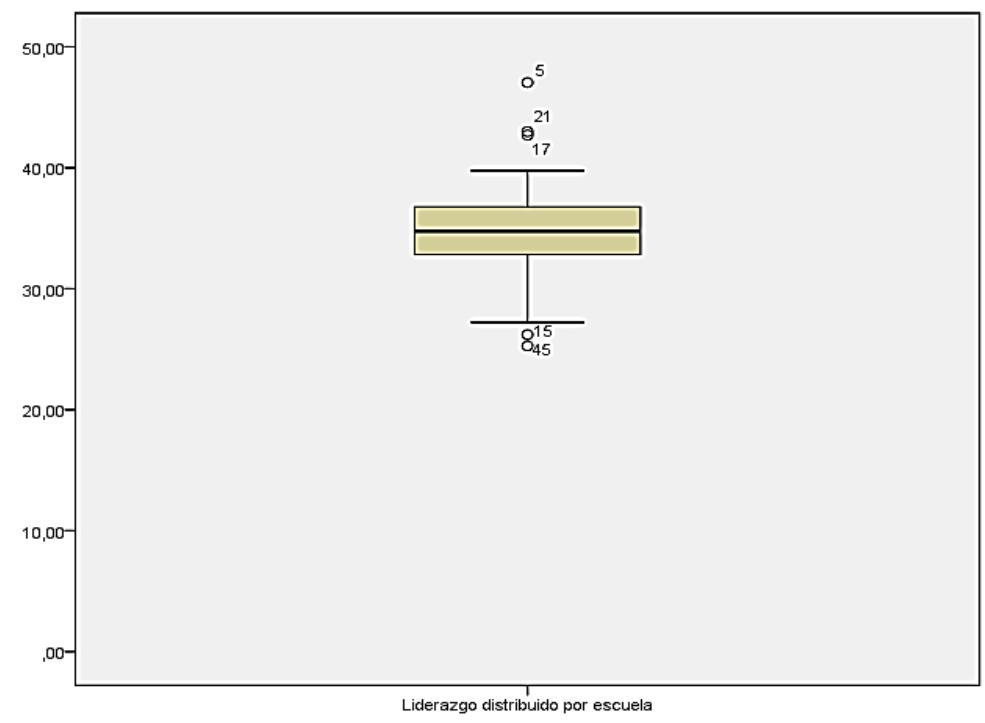

Gráfico 2. Representación de la mediana del liderazgo distribuido en las escuelas

Sobre la base de estos datos, se pudo afirmar que en general los directivos tendían a interactuar con los líderes formales, informales, los seguidores y los diferentes actores pertenecientes al contexto de la escuela, para propiciar la distribución social de la influencia en el grupo, y delegaban las funciones de dirección, orientación, la toma de decisiones, las tareas y los compromisos que guiaban y daban sentido a la organización.

El mínimo puntaje fue de 25.28 , por lo tanto, no hubo escuelas con puntajes bajos o muy bajos. El valor máximo fue 47,04, y se ubicó en la categoría muy alta. El grupo de escuelas fue muy homogéneo, en cuanto a liderazgo distribuido. 
$\mathrm{Al}$ analizar la frecuencia y porcentajes en la categoría de liderazgo distribuido sobre la base de los datos de la Tabla 2 se observó que el $6.1 \%$ de los directivos se ubicó en la categoría media de liderazgo distribuido. Seguidamente el $43.50 \%$ de los directivos obtuvieron puntajes altos, y un $4.6 \%$ muy alto. No se observaron valores bajos.

Tabla 2. Frecuencias y porcentajes en la categoría liderazgo distribuido

\begin{tabular}{lllll}
\hline Categoría & Frecuencia & Porcentaje & $\begin{array}{l}\text { Porcentaje } \\
\text { válido }\end{array}$ & $\begin{array}{l}\text { Porcentaje } \\
\text { acumulado }\end{array}$ \\
\hline Medio & 8 & 6,1 & 11,3 & 11,3 \\
Alto & 57 & 43,5 & 80,3 & 91,5 \\
Muy alto & 6 & 4,6 & 8,5 & 100,0 \\
\hline Total & 71 & 54,2 & 100,0 & \\
\hline
\end{tabular}

Los resultados obtenidos mostraron que el liderazgo distribuido se ha difundido en las escuelas públicas, más de lo que pudiera suponerse y es aceptado por la mayoría de los directores de las 71 escuelas estudiadas en Madrid. La mayoría de los directores estuvieron de acuerdo con las prácticas de LD. Además, al comparar el LD de los directores con los jefes de estudio, se encontró que ambos directivos hacían énfasis en la interacción con los maestros, colaboradores o empleados, con lo cual se reconocía la labor de todos los individuos al contribuir en la práctica, en la toma de decisiones y en la delegación de responsabilidades y autoridad, de allí que el rediseño de la organización requirió de un proceso de toma de decisiones compartidas para su buen funcionamiento.

$\mathrm{Al}$ analizar las diferentes dimensiones del liderazgo distribuido (Tabla 3), se observó que la dimensión con mayor mediana es prácticas de liderazgo distribuido con 37,50 puntos sobre una escala de 50 .

Tabla 3. Medianas de las dimensiones de liderazgo distribuido

\begin{tabular}{|c|c|c|c|c|c|}
\hline & & PLD & DC & DPCT & MVMC \\
\hline Mediana & & 34,76 & 32,58 & 33,77 & 36,36 \\
\hline Mínimo & & 25,28 & 23,21 & 24,68 & 22,73 \\
\hline Máximo & & 47,04 & 49,11 & 47,04 & 48,46 \\
\hline \multirow[t]{3}{*}{ Percentiles } & 25 & 32,79 & 28,12 & 31,25 & 31,81 \\
\hline & 50 & 34.76 & 32,58 & 33,77 & 36.36 \\
\hline & 75 & 36,94 & 35,71 & 37,05 & 41,47 \\
\hline
\end{tabular}




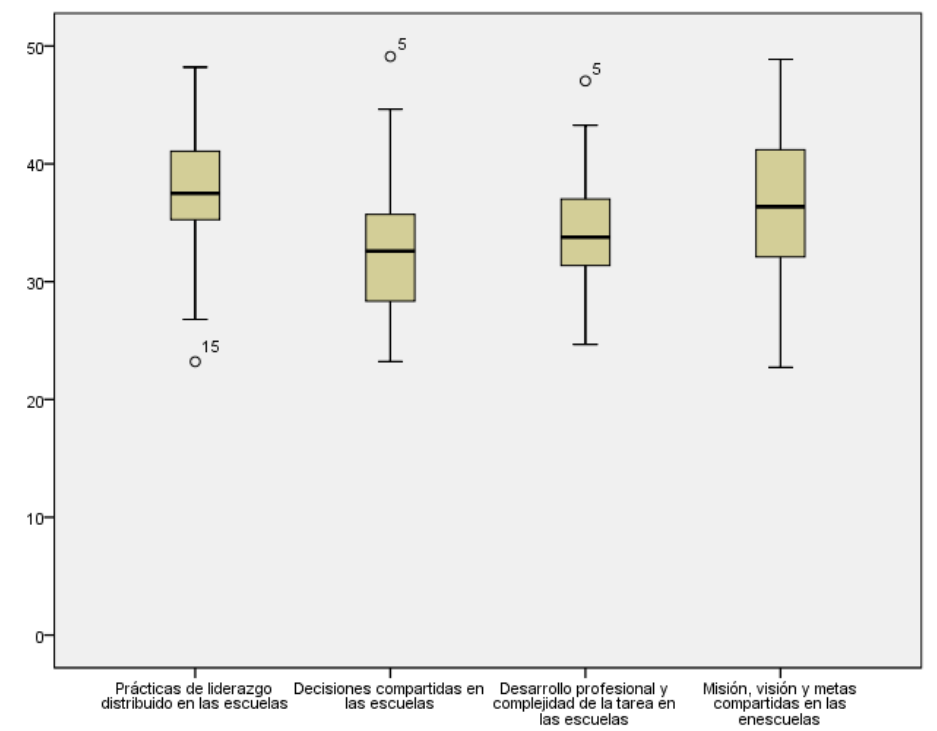

Gráfico 3. Representación de las medianas de las dimensiones liderazgo distribuido

En segundo lugar, el grupo de directivos obtuvo una mediana de 36.36 puntos sobre una escala de 50 en la dimensión misión, visión y metas compartidas. En tercer lugar, se observó la dimensión desarrollo profesional y complejidad de la tarea en las escuelas con una mediana de 33.77 puntos sobre una escala de 50. En cuarto y último lugar se posicionó la dimensión decisiones comprartidas en las escuelas con una mediana de 32.59 puntos sobre una escala de 50 .

$\mathrm{Al}$ analizar los datos de la Tabla 4 y el gráfico 4, correspondientes a la dimensión prácticas de liderazgo distribuido, se observó que era en esta dimensión donde mejor se desempeñaban los directivos en general. La mediana se colocó en 37.50 puntos sobre una escala de 50 , en la categoría alta. Significó que los directivos se esforzaban por delegar el trabajo, y consideraban que el trabajo conjunto podía aportar aprendizajes para la organización, además, los directivos asumieron que las formas de liderazgo más participativas funcionaban mejor. La distribución no fue simétrica ya que, aunque la mediana estaba en la mitad de la caja, toda la caja se corrió hacia la parte superior.

Tabla 4. Mediana de la dimensión práctica de liderazgo distribuido

\begin{tabular}{lll}
\hline Mediana & & $\mathbf{3 7 , 5 0}$ \\
\hline Mínimo & & 23,21 \\
Máximo & & 48,21 \\
Percentiles & 25 & 34,82 \\
& 50 & 37,50 \\
& 75 & 41,07 \\
\hline
\end{tabular}




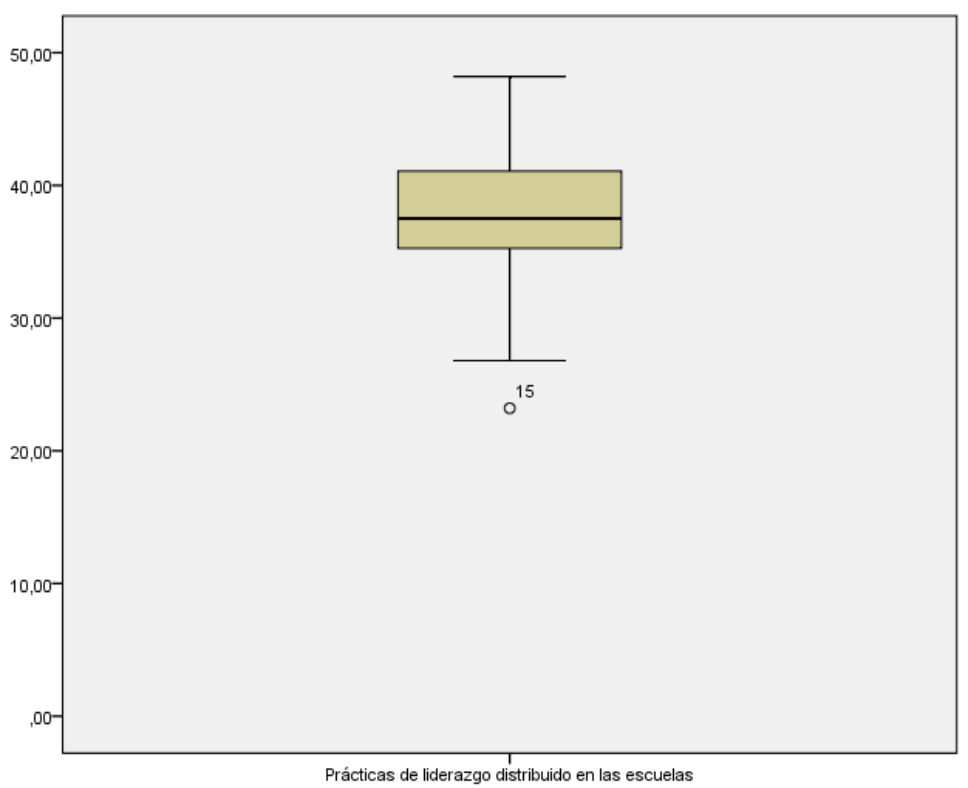

Gráfico 4. Representación de la mediana de la dimensión práctica de liderazgo distribuido

El hecho de que la mediana de la dimensión prácticas de liderazgo distribuido se ubicara en la categoría alta, significaba que estos directivos tendían, en general, a distribuir las tareas en función de las competencias y habilidades de quienes trabajaban con ellos, se inclinaban a delegar aspectos de su trabajo como directores y fomentaban el surgimiento de otros líderes dentro de la organización, como una forma de abordar tareas complejas y lograr las metas institucionales.

Fue así como las prácticas de liderazgo distribuido pudieron mejorar varios aspectos como son: la motivación del personal, una mayor capacidad en el equipo de trabajo, la posibilidad de diseminar un carácter más democrático en toda la escuela y una mayor flexibilidad.

En cuanto a la segunda dimensión, misión, visión y metas compartidas. $\mathrm{Al}$ analizar los datos de la Tabla 5 se observó la ubicación de la mediana en 36,36 puntos sobre una escala de 50 , en la categoría alta, lo que significó que en general los directivos promovían el compromiso, por parte de todos los miembros de la institución, hacia metas comunes orientadas hacia la misión y la visión de la escuela, y los directivos trabajaban en la creación de valores compartidos. En relación con la dispersión de los datos, el grupo de directores era relativamente homogéneo en esta dimensión.

Tabla 5. Mediana de la dimensión misión, visión y metas compartidas

\begin{tabular}{lll}
\hline Mediana & & $\mathbf{3 6 , 3 6}$ \\
Mínimo & & 22,73 \\
Máximo & & 48,86 \\
Percentiles & 25 & 31,81 \\
& 50 & 36,36 \\
& 75 & 41,47 \\
\hline
\end{tabular}




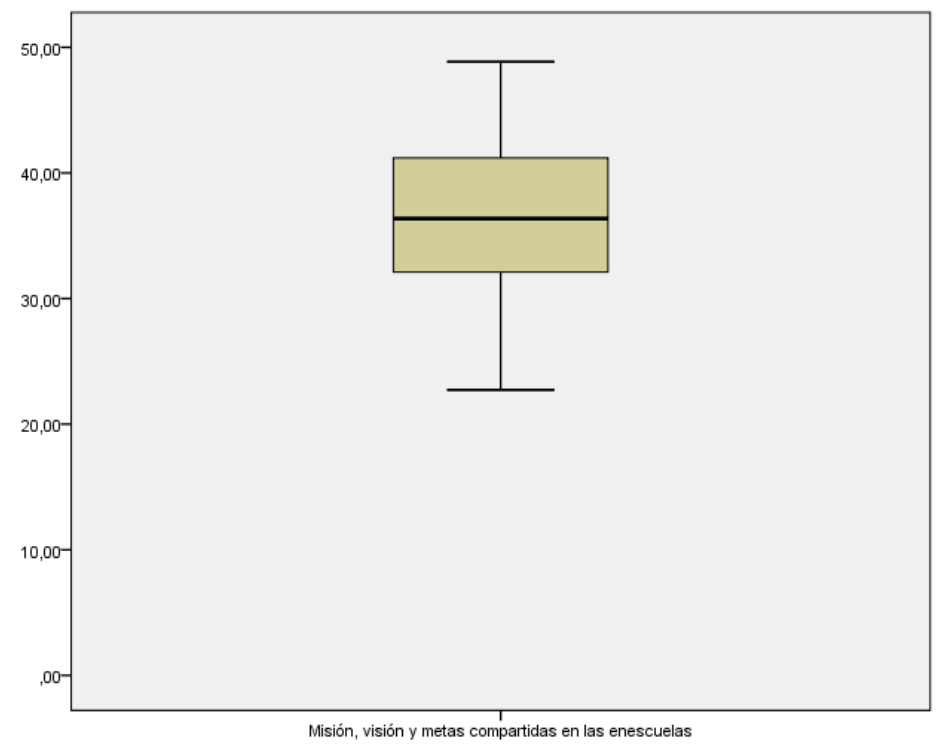

Gráfico 5. Representación de la mediana de la dimensión misión, visión y metas compartidas

Esta dimensión abordó aspectos críticos del liderazgo distribuido que consistían en ayudar al grupo a desarrollar un conocimiento compartido de la organización, de las actividades y objetivos, con el fin de crear un sentido de propósito o visión.

La importancia de que los líderes distribuidos se orienten a fijar rumbos reside en que se basan en las teorías de la motivación humana para dichos objetivos. De acuerdo con las teorías ya estudiadas, las personas se sienten motivadas por los objetivos que consideran personalmente atractivos y desafiantes, pero que, al mismo tiempo, sienten que pueden alcanzar. Estos objetivos les permiten a los docentes, directivos y personal en general darle un sentido a su trabajo, lo que les posibilita desarrollar un sentido de identidad propio dentro de su contexto de trabajo en la escuela.

Las experiencias específicas que contribuyen a brindar una orientación y que frecuentemente se mencionan son: Identificar y articular una visión y promover la aceptación de los objetivos grupales. Al fomentar la aceptación de metas grupales, los líderes ayudan a fijar un rumbo, y alientan al equipo educativo a desarrollar metas compartidas. Las personas se sienten motivadas por metas que consideran convin-centes, desafiantes y factibles. La existencia de esas metas ayuda a las personas a entender la lógica de la escuela y a elaborar una identidad dentro de su contexto laboral. Las metas compartidas también permiten orientar la actividad organizacional en una dirección común para lograr el máximo im-pacto.

Un aspecto relevante identificado en esta dimensión fue el hecho de que los directivos estaban focalizados en proporcionar apoyo individual y modelar las prácticas y los valores, pero una de las debilidades estaba en que no todo el personal compartía la filosofía del colegio.

La tercera dimensión, desarrollo profesional, complejidad de la tarea e incentivos, se ubicó en un tercer lugar y su mediana se colocó en 33,77 puntos sobre una escala de 50, en la categoría alta (Tabla 6), lo que significó que las acciones de liderazgo contribuyeron al desarrollo de las personas para avanzar productivamente en la institución. Además, esta dimensión incluyó la 
planificación del desarrollo profesional del equipo, a través de asistencia a seminarios, cursos, talleres, etc., y se apoyó la asignación de los recursos necesarios para impulsar los esfuerzos de mejoramiento escolar.

Al contrastar los resultados de los directores y los jefes de estudio, se observó que ambos incorporaban la reflexión y el diálogo para delegar, orientar, aprovechar y mejorar las habilidades y conocimientos de todos los maestros, y crear una cultura común positiva y eficaz, y además se valían de un sistema de metas e incentivos. Para estos directivos, el desarrollo profesional de los docentes se convirtió en una característica clave de las escuelas de calidad.

Tabla 6. Mediana de la dimensión desarrollo profesional, complejidad de la tarea e incentivos

\begin{tabular}{lll}
\hline & & DPCT \\
\hline Mediana & & $\mathbf{3 3 , 7 7}$ \\
Mínimo & & 24,68 \\
Máximo & & 47,04 \\
Percentiles & 25 & 31,25 \\
& 50 & 33,77 \\
& 75 & 37,05 \\
\hline
\end{tabular}

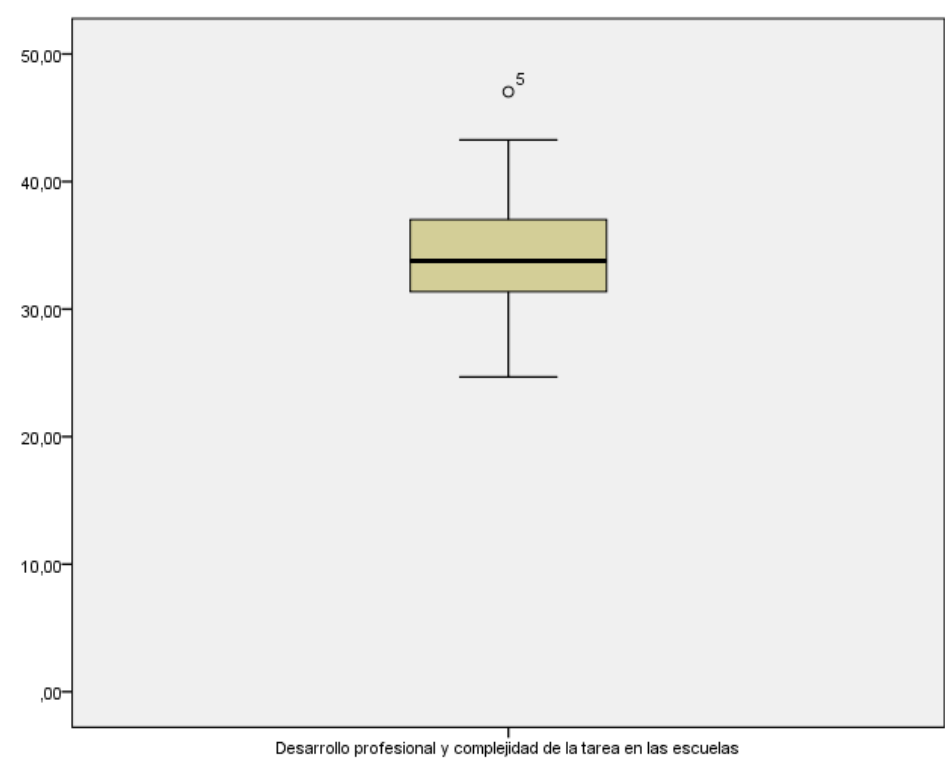

Gráfico 6. Representación de la mediana de la dimensión desarrollo profesional, complejidad de la tarea e incentivos

Un $74.6 \%$ de los directores y un $60 \%$ de los jefes(as) de estudio, apoyaron la comunicación para beneficiar y mejorar las competencias y conocimientos de todos los maestros, y crear un clima común positivo y eficaz. Se trabajó formalmente en el currículo escolar de forma consistente y coherente entre materias y niveles, además de en el sistema de metas e incentivos.

La cuarta y última dimensión que se analizó fue decisiones compartidas. Esta dimensión ocupó el cuarto lugar con un valor de la mediana de 32.58 puntos sobre una escala de 50 (Tabla 7), ubicada en la categoría 
alta, esto significó que los directivos tendían a trabajar en función de la cooperación en lugar de la competencia, y a promover una visión holística de la organización. El directivo creaba las condiciones para que los miembros de la organización discutieran y se ayudaran de una forma espontánea a la hora de resolver cualquier problema.

Tabla 7. Mediana de la dimensión decisiones compartidas

\begin{tabular}{lll}
\hline & & DC \\
\hline Mediana & & $\mathbf{3 2 , 5 8}$ \\
Mínimo & & 23,21 \\
Máximo & & 49,11 \\
Percentiles & 25 & 28,12 \\
& 50 & 32,58 \\
& 75 & 35,71 \\
\hline
\end{tabular}

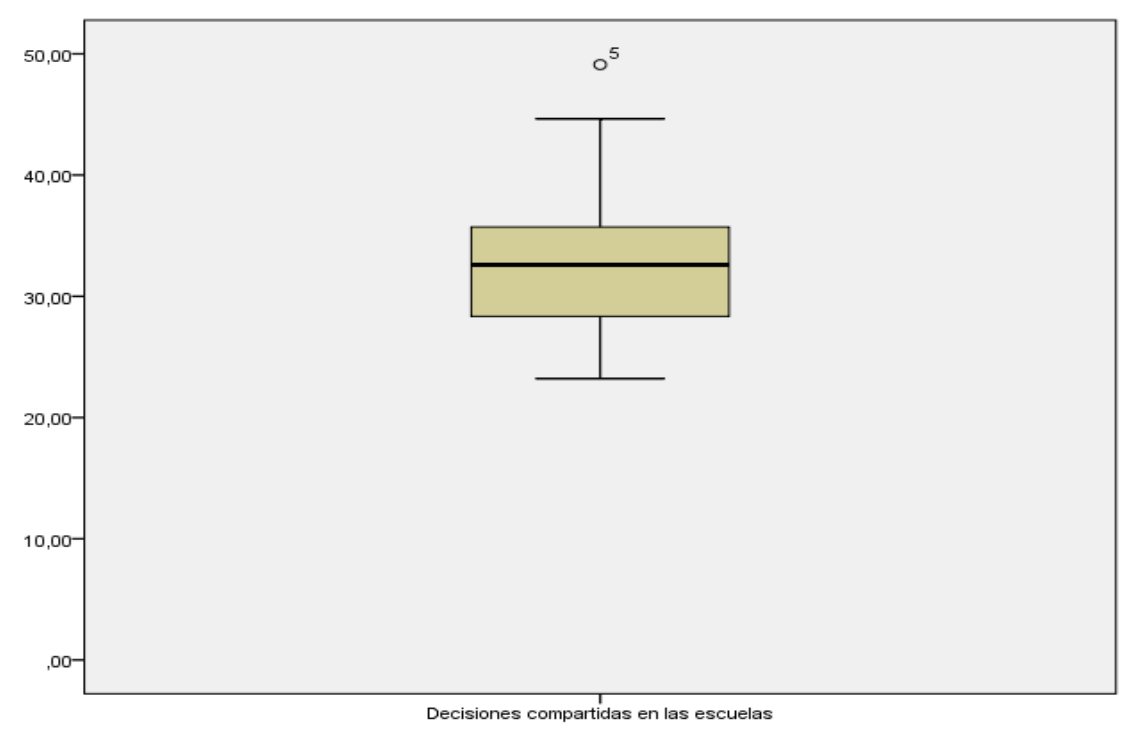

Gráfico 7. Representación de la mediana de la dimensión decisiones compartidas

Las decisiones compartidas se asocian y se definen como elementos medulares que determinan las respuestas de liderazgo productivo, las formas de liderazgo más participativas, compartidas o distribuidas, funcionan mejor en respuesta a tareas relativamente complejas y son productivas cuando las tareas a realizar son relativamente sencillas. La dimensión decisiones comprartidas que se observó en las escuelas se relacionaba con:
- Distribución colaborativa: donde dos o más personas trabajaban juntas en la misma hora y lugar para ejecutar la misma rutina de liderazgo.

- Distribución colectiva: donde dos o más personas trabajaban por separado pero interdependiente para promulgar una rutina de liderazgo.

- Distribución coordinada: donde dos o más personas trabajaban en secuencia con el fin de completar un liderazgo de rutina.

El 95\%de los directores y 97\%, de los jefes de estudio manifestaron tener como propósito 
el rediseño de las estructuras de la escuela con el fin de fomentar la participación y hacerla más flexible. Al hacer esto los logros se podrán asociar de manera general con el fortalecimiento de la cultura escolar. Otra forma de rediseñar las estructuras organizacionales es a través de cambios en los equipos de trabajo, tareas asignadas, la calendarización, el diseño del tiempo, los procedimientos operativos rutinarios y el uso de tecnologías más otros recursos materiales; elementos estos que orientan los cambios estructurales hacia el establecimiento de condiciones positivas para los procesos de enseñanza-aprendizaje, pero que al mismo tiempo, conjuntamente con los directores(as), pueden dificultar o facilitar el rendimiento personal y el cumplimiento de metas organizacionales.

\section{Discusión}

\section{Análisis en la sustentación teórica sobre el liderazgo para los directivos en los planteles educativos}

Yukl y Tabbler (2002) consideran que el liderazgo es un proceso de influencia capaz de afectar la elección de los objetivos de la organización, el desarrollo de actividades para lograr estos objetivos, la motivación de los seguidores a cumplir los objetivos, el mantenimiento de relaciones de cooperación y la obtención de apoyo de las personas fuera del grupo u organización.

El enfoque teórico del liderazgo distribuido se ha construido sobre dos principios el liderazgo compartido (Senge, 2006) y el participativo (Immegart, 1996), los fundamentos que se estudiaran más adelante, se originan básicamente a partir de los principios de dos teorías, la cognición distribuida (Hutchins, 2006) y de la actividad (Engerström, 2000, Engeström y Blackler, 2005).
Calder (1977) define el liderazgo distribuido como una condición específicamente atribuida a un individuo o un conjunto de individuos distintos que en grupos pequeños, actúan en armonía con los demás miembros, departamentos o unidades de la organización.

Autores como Gronn (2002) y Spillane (2005) han profundizado en el estudio de la distribución del poder en las estructuras educativas, obteniendo un concepto focalizado más hacía la práctica del liderazgo que en el rol tradicional de perfiles y patrones directivos o de jefaturas, sus antecedentes y consecuencias. El líder, desde un contexto distribuido, se destaca porque no se sitúa solo en la figura directiva, o de poder, sino que se distribuye o extiende hacia el trabajo de múltiples líderes que ejercen una influencia en las prácticas de enseñanza en la organización escolar. Esto se traduce en el liderazgo como función, se apoyaría en quienes ejercen la dirección, jefaturas intermedias, coordinaciones, profesorado tutor y de aula (Crawford, 2005).

Gronn (2002), describe el liderazgo distribuido, como nuevas formas de hacer para las direcciones escolares, donde interaccionan rasgos y comportamientos del director con la estructura y sistema de la organización escolar. Este estilo de liderazgo está sustentado por una filosofía colectiva, la cual genera una mayor autonomía en las prácticas de liderazgo distribuido en los centros educativos, genera para el propio centro, donde los agentes escolares juegan un papel importante para el funcionamiento organizativo. En el trabajo de Gronn (2000) se sugiere que el LD, es una propiedad emergente de un grupo o una red de individuos que interactúan. Aquí el liderazgo es una forma de acción concertada, que se localiza en la dinámica adicional que se produce cuando las personas trabajan juntas o que es el producto de la acción conjunta. 
Para Gronn (2000) el liderazgo distribuido se puede manifestar de tres formas:

- La colaboración espontánea. De vez en cuando, grupos de personas con diferentes destrezas y conocimientos, provenientes de diferentes niveles de la organización, se unen para poner en común su pericia y regularizar sus conductas durante una tarea, y luego se separan.

- La distribución concertada, en la medida en que dos o más miembros de la organización llegan a apoyarse mutuamente y desarrollan relaciones de trabajo cercanas y el liderazgo se manifiesta en el espacio de los roles compartidos que abarca la relación.

- Por último, la práctica institucionalizada de Gronn (2002), para quien los comités y los equipos representan su forma más obvia, e incluye a las estructuras formalizadas que surgen por designio o a través de una adaptación menos sistemática.

El liderazgo distribuido se centra en la interacción entre los directivos, maestros, colaboradores o empleados, en la cual se reconoce la labor de todos los individuos al contribuir en la práctica en la toma de decisiones y en la delegación de responsabilidades y autoridad, aun cuando no son formalmente designados o definidos como dirigentes, y es fundamental en la configuración del sistema y de la organiza-ción, ya que el rediseño de la organización requiere de un proceso de toma de decisiones compartidas

Hoy por hoy, el liderazgo distribuido se considera un concepto multidimensional con conceptos superpuestos de otros liderazgos (Ritchie y Woods, 2007 y López-Yánez y
Lavié, 2010). Las cuatro dimensiones del liderazgo distribuid son:

1. Visión, misión y metas compartidas (VMMC): es el aspecto del liderazgo distribuido que implica que todos los miembros de la escuela están comprometidos, con las metas de la institución en un sentido de propósito y visión a largo plazo; se considera que la escuela cuenta con un conjunto de valores compartidos que conducen los esfuerzos, fomentan la confianza y el compromiso entre maestros, directivos, personal administrativo y comunidad educativa. Hay un firme compromiso con los objetivos de la organización en su conjunto y se comparte con todo el personal la filosofía del colegio y una visión futurista de la escuela; la escuela es sensible a las necesidades de los alumnos, padres y comunidad y se genera apoyo para establecer y comunicar al grupo claramente el rumbo de la institución.

\section{Prácticas de liderazgo distribuido (PLD):} se delega el trabajo, y el director considera que la suma de las acciones representa más que las partes, pues el trabajo participativo se traduce en un aprendizaje para la organización. Los directivos asumen que las formas de liderazgo más participativas funcionan mejor y permiten acceder a los conocimientos colectivos de la organización, así como manejar procesos participativos para resolver de manera productiva los conflictos, lo cual requiere concertar y regularizar las relaciones informales para mejorar los acuerdos ya existentes.

3. Desarrollo profesional de los maestros y complejidad de la tarea (DPMCT): se relaciona con la reflexión y el diálogo para delegar, orientar, aprovechar y mejorar las habilidades y conocimientos de todos 
los docentes, y crear una cultura común positiva y eficaz, así como proporcionar un currículo escolar consistente y coherente entre materias y niveles, además de un sistema de metas e incentivos. Las acciones de liderazgo contribuyen al desarrollo de las personas para avanzar productivamente en la institución. Se practica la dirección y el liderazgo como reflexión de la cultura del colegio, de la ética y las tradiciones. Además, esta dimensión incluye la planificación del desarrollo profesional del equipo, a través de asistencia a seminarios, cursos, talleres, etc., y se apoya la asignación de los recursos necesarios para impulsar los esfuerzos de mejoramiento escolar.

4. Decisiones compartidas de la organización (DC): se trabaja sobre la base de la cooperación en vez de la competencia, para promover la productividad dentro de la escuela, con una visión holística de la organización y con las prácticas profesionales flexibles. Los miembros de la organización discuten y se ayudan de una forma espontánea a la hora de resolver cualquier problema, por medio de acuerdos para modificar ciertas estructuras organizacionales, y a través de la participación de los padres, a fin de que tomen decisiones sobre la enseñanza y el aprendizaje de sus hijos en la escuela.

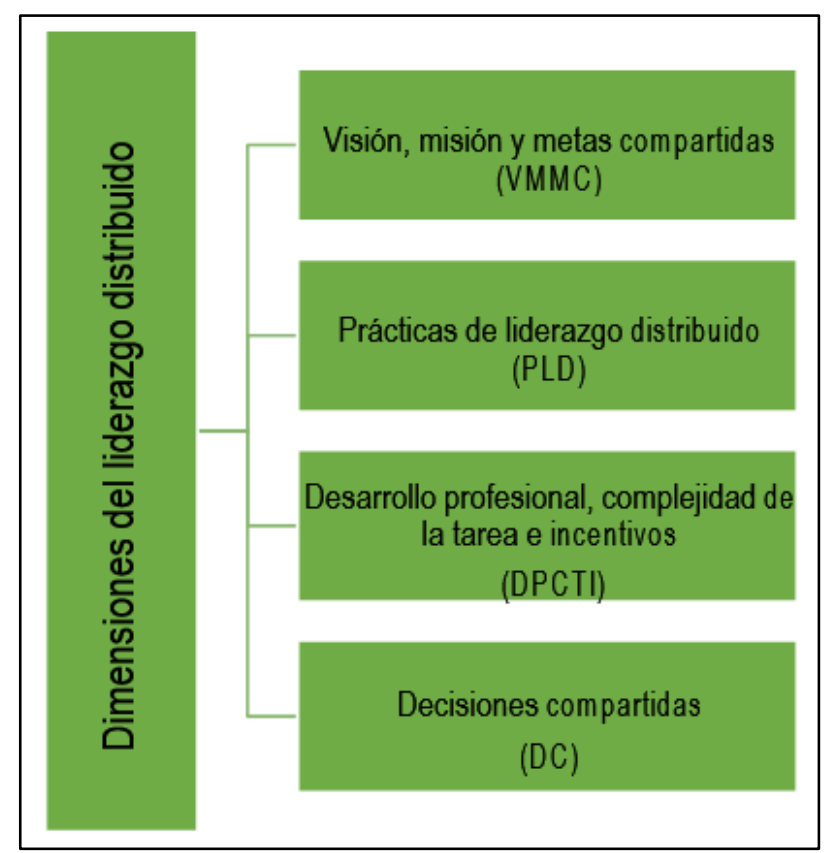

Gráfico 2. Dimensiones del liderazgo distribuido

Trabajar en los centros escolares desde un modelo así, supone que el liderazgo puede proceder de cualquier lugar de la organización. Su puesta en práctica se relaciona con dinámicas de trabajo que grupos y agentes despliegan en contextos organizativos específicos (López y Lavié,
2010). Para este fin es importante estudiar el desarrollo de la dirección escolar en España y el estilo de liderazgo en los centros escolares. 


\section{CONCLUSIONES}

Con respecto al objetivo general del presente estudio que fue describir las prácticas y percepción del liderazgo distribuido de los centros primarios públicos de Madrid Capital, en presencia de un conjunto de características propias y de las condiciones particulares de las escuelas; Los resultados globales obtenidos mostraron que el liderazgo distribuido se ha difundido, más de lo que pudiera suponerse y es aceptado por la mayoría de los directivos de las 71 escuelas estudiadas en Madrid.

Los directivos tendían a interactuar con los líderes formales, informales, los seguidores y los diferentes actores perte-necientes al contexto de la escuela, para propiciar la distribución social de la influencia en el grupo, y delegaban las funciones de dirección, orientación, la toma de decisiones, las tareas y los compromisos que guiaban y daban sentido a la organización.

Al comparar el liderazgo distribuido en los dos grupos, el de directores y el de jefes de estudio de las 71 escuelas públicas primarias, se concluyó que ambos hacían énfasis en la interacción con los maestros, colaboradores y empleados. Se reconocía la labor de todos los individuos al contribuir en la práctica, en la toma de decisiones y en la delegación de responsabilidades y autoridad; igualmente con relación al rediseño de la organización se observó que este requería de un proceso de toma de decisiones compartidas para su buen funcionamiento. Sin embargo, los jefes(as) de estudio de las escuelas, distribuían el tiempo diario en actividades de gestión tales como disciplina escolar, gestión de instalaciones, horarios, notas, exámenes, entre otras. Estas actividades estaban directamente ligadas a la instrucción, así como al seguimiento a estudiantes y docentes, planificación, participación en comité y reuniones relacionadas con la docencia.
En la dimensión práctica de liderazgo distribuido (PLD), se encontró que un porcentaje alto de directivos estaba de acuerdo con estas prácticas. Al comparar las cuatro dimensiones, se observó que era en esta dimensión donde mejor se desempeñaban los directivos en general. Ellos tendían a distribuir las tareas en función de las competencias y habilidades de quienes trabajaban con ellos, se inclinaban a delegar aspectos de su trabajo como directores y fomentaban el surgimiento de otros líderes dentro de la organización, como una forma de abordar tareas complejas y lograr las metas institucionales.

Estos resultados evidenciaron que las escuelas públicas primarias españolas analizadas, se encontraban muy lejos de los modelos tradicionales, en donde el equipo de liderazgo se constituye exclusivamente por personal docente calificado y por lo regular incluye al director apoyado por los maestros.

Igualmente se concluyó sobre los beneficios de las prácticas de liderazgo distribuido, las cuales pudieron mejorar varios aspectos como fueron: la motivación del personal, una mayor capacidad en el equipo de trabajo, la posibilidad de diseminar un carácter más democrático en toda la escuela y una mayor flexibilidad, mediante el trabajo colaborativo con actores comprometidos que sean capaces de adoptar sugerencias generales, para retomar las fortalezas y superar las debilidades de los liderazgos individuales.

Al contrastar los resultados se observó que los jefes de estudio en general, realizaban otras labores además de distribuir las tareas a las personas que trabajaban con ellos, pero no delegaban aspectos de su trabajo, lo que implicaba que ejercían, menos que los directores, el liderazgo distribuido. Los jefes de estudio consideraban formas de liderazgo más participativas, y accedían a los conoci- 
mientos colectivos de la escuela, esta conclusión resultó al observar la relación directa que tenían con los maestros $\mathrm{y}$ familiares, a diferencia de los directores.

El aspecto peor valorado fue el trabajo de dos o más líderes que se hacía separada e interdependientemente. Los directores no estaban de acuerdo con el trabajo de dos o más líderes, ni con la colaboración espontánea, que se refiere a grupos de personas con diferentes destrezas y conocimientos, provenientes de diferentes niveles de la organización; que se unían, durante una tarea, para poner en común su pericia y regularizar sus conductas ejerciendo liderazgos propios, y luego se separaban.

En la segunda dimensión misión, visión y metas compartidas (MVMC) se evidenció, que un porcentaje alto de directores estaban dispuestos a ayudar al grupo en el desarrollo del conocimiento compartido de la organización, de las actividades y objetivos, con el fin de crear un sentido de propósito o visión. Esta dimensión abordó aspectos críticos del liderazgo distribuido que podían permitir a los docentes, directivos y personal en general darle sentido a su trabajo, lo que les posibilitó a desarrollar una conciencia de identidad dentro de la escuela.

El grupo de los directores y los jefes de estudio manifestaron apoyo a sus equipos con respeto y consideración por sus sentímientos y necesidades personales.

Al contrastar la posición de los directores(as) con relación a los jefes de estudio en esta dimensión, se encontró que los jefes de estudio poseían un firme compromiso con las metas compartidas de la organización en su conjunto y compartían con todo el personal la filosofía del colegio y una visión a largo plazo de la escuela.

El desarrollo y el compartir conocimiento dentro de la organización, resultó ser el ítem más valorado por directores y jefes de estudio. Los líderes distribuidos ayudaban a identificar nuevas oportunidades para la escuela que podían resultar inspiradoras. La mayor debilidad fue que no todo el personal compartía la filosofía del colegio, ni la visión a largo plazo de la escuela.

En relación con la tercera dimensión desarrollo profesional, complejidad de la tarea e incentivos (DPCTI), la percepción de la dimensión para estos directivos fue entendida como variable clave que determina las respuestas de liderazgo productivas, así como el uso de formas de liderazgo más participativas, compartidas o distribuidas. Esto implicó que funcionaran mejor en respuesta a tareas relativamente complejas y no eran productivas con tareas relativamente sencillas. Por otro lado, el desarrollo profesional de los docentes se convirtió en una característica clave de las escuelas de calidad.

Los directores y jefes de estudio estuvieron de acuerdo con una escuela en la que exista preocupación por parte de toda la comunidad, pero fundamentalmente de los docentes, por seguir aprendiendo y mejorando, y expresaron que esa también era la escuela donde los alumnos aprenden más.

El ítem con mayor porcentaje de aceptación fue que la reflexión y diálogo eran fundamento para una buena toma de decisiones. Este ítem midió la disposición y la capacidad de los directivos(as) para tomar control de sus ideas a la hora de tomar decisiones.

El peor valorado fue la existencia de algún sistema de metas e incentivos que operara en la escuela para los profesores. Los directores $y$ jefes de estudio pensaban que este sistema no existía, a pesar de reconocer que los sistemas de incentivos son indispensables para promover un clima 
académico que establezca expectativas y elevados estándares positivos de conducta.

Por último, en la cuarta dimensión decisiones compartidas, se evidenció que, en lugar de la competencia, para promover la productividad se fomentaba, dentro de la escuela, la cooperación con una visión holística de la organización y con prácticas profesionales flexibles.

El aspecto más valorado fue tener como propósito el rediseño de las estructuras de la escuela con el fin de fomentar la participación y hacerla más flexible.

\section{REFERENCIAS}

Barber, M. y Mourshed, M. (2007). How the world's best-performing schools systems come out on top. Londres: McKinsey.

Bolden, R. (2011). Distributed leadership in organizations: A review of theory and research. International Journal of Management Reviews, 13(3), 251-269.

Calder, B. (1977). An attribution theory of leadership. NuevaDirections in Organizational Behavior, 179(1), 204-225.

Crawford, M. (2005). Distributed leadership and headship: A paradoxical relationship?

Day, C., Sammons, P., Leithwood, K., Hopkins, D. Gu, Q. y Brown, E., with Ahtaridou, E. (2011). Successful School Leadership: Linking with Learning and Achievement. Maidenhead: McGraw Hill Open University Press.

Dinham, S., Anderson, M., Caldwell, B. y Weldon, P. (2011). Breakthroughs in school leadership development in Australia. School Leadership y Manage-ment: Formerly School Organisation, 31(2), 139154.

Engeström, Y. (2000). Activity theory as a framework for analyzing and redesigning work. Ergonomics, 43(7), 960-974.
Engeström, Y. y Blackler, F. (2005). On the life of the object. Organization, 12(3), 307-330.

Gairín, J y Muñoz, J (2013). La organización escolar como campo de estudio e investigación. Revista anthropos: Huellas del conocimiento 1(238), 11-27.

Gronn, P. (2000). Distributed properties a new architecture for leadership. Educational Management Administration y Leadership, 28(3), 317-338.

Gronn, P. (2002). Distributed leadership as a unit of analysis. Leadership Quarterly, 13(4), 423-451.

Gronn, P. (2006). The significance of distributed leadership. Educational Leadership Research, 63 (7), 160-172.

Gronn, P. y Hamilton, A. (2004). A bit more life in the leadership: Co-principal ship as distributed leadership practice. Leadership and Policy in Schools, 3(1), 3-35.

Gunter, H. y Fitzgerald, T. (2008). The future of leadership research? School Leadership

Harris, A. (2004). Teacher leadership and distributed leadership: An exploration of the literature in leading and managing. Leading and Managing, 10(2), 1-10.

Harris, A. y Spillane, J. (2008). Distributed leadership through the looking glass.

Management in Education, 22(1), 31-34.

Harris, A., Day, C., Hopkins, D., Hadfield, M., Hargreaves, A. y Chapman, C. (2013).

Hutchins, E. (2006). The distributed cognition perspective on human interaction. Roots of human sociality: Culture, cognition and interaction, 1, 375.

Immegart, G. L. (1996). Dirección participativa: realidades prácticas, estratégicas y éticas. In Dirección participativa y evaluación de centros (pp. 361-376). Universidad de Deusto; Deustuko Unibertsitatea.

Leithwood, K. (2009). Cómo liderar nuestras 
escuelas: aportes desde la investigación. Santiago de Chile: Fundación Chile.

López-Yáñez, J. y Lavié, J. (2010). Liderazgo para sostener procesos de innovación. Profesorado: Revista de Curriculum y Formación del Profesorado, 14(1), 71-92.

MacBeath, J., Oduro, G. y Waterhouse, J. (2004). Distributed leadership in action: A study of current practice in schools.

Nottingham: National College for School Leadership. National College for School Leadership, NCSL (2008)

Oduro, G. (2004). Distributed leadership in schools: What English headteachers say about the" pull" and" push" factors. Comunicación presentada en la conferencia anual de la British Educational Research Association, Man-chester.

Pont, B., Nusche, D., Moorman, H. y Hopkins, D. (2008). Mejorar el liderazgo escolar, volumen 1 política y práctica. Paris: OECD Publishing

Ritchie, R y Woods, P. (2007). Degrees of distribution: Towards an understanding of variations in the nature of distributed leadership in schools. School Leadership and Management, 27(4), 363-381

Senge, P. (2006). The fifth discipline: The art and practice of the learning Organization. Nueva York: Random House Digital, Inc.

Sloan, T. (2013). Distributed leadership and organizational change: Implementation of a teaching performance measure. The New Educator, 9(1), 29-53.

Spillane, J. (2005). Distributed leadership. The Educational Forum, 69(2), 143-150.

Spillane, J. (2006). Distributed leadership. San Francisco: John Wiley y Sons.

Spillane, J., Halverson, R. y Diamond, J. (2004). Towards a theory of leadership practice: A distributed perspective. Journal of Curriculum Studies, 36(1), 3-34.

Yukl, G. y Tabbler, T. (2002). A hierarchical taxonomy of leadership behavior: Integrating a half century of behavior research. Journal of Leadership $y$ Organizational Studies, 9(1), 15-32 